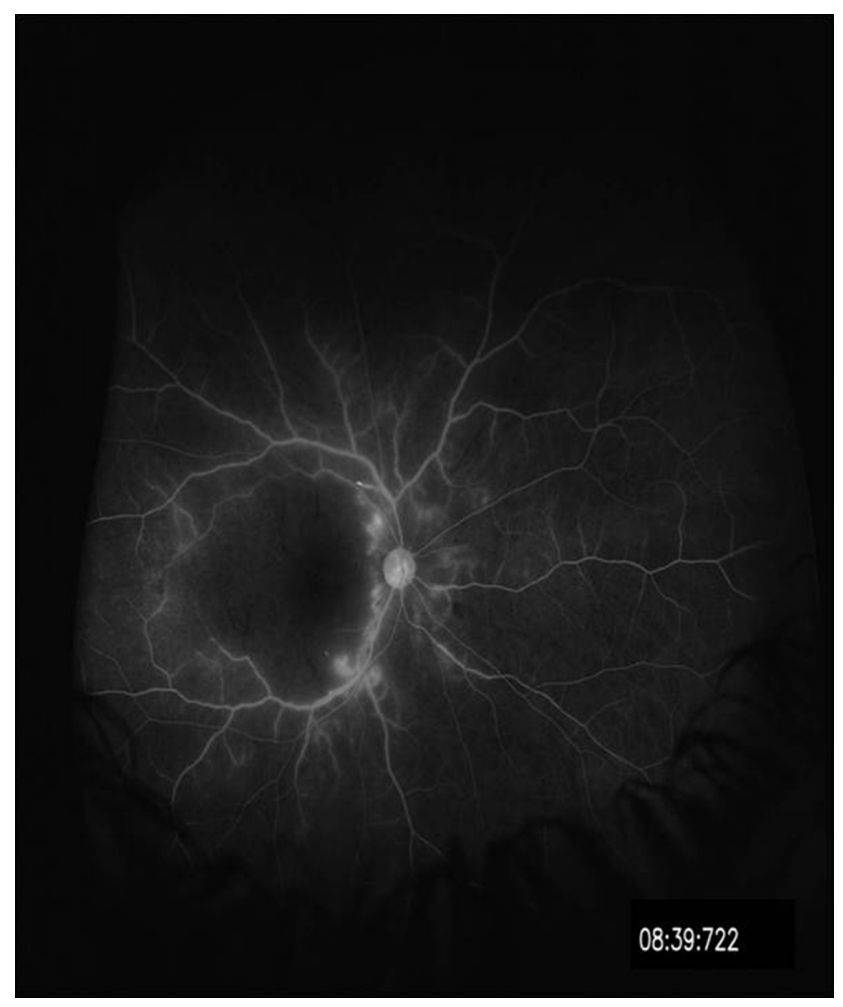

Abstract 134 Figure 1

done that revealed capillary 'drop-out', vessel wall staining and leakage of dye. (Figure 1) He had antinuclear antibodies (4+ speckled pattern). A diagnosis of SLE with isolated retinal involvement (vasculopathy) was considered. In view of severely impaired visual acuity, he was managed with injection cyclophosphamide (followed by maintenance mycophenolate mofetil) and pulse methylprednisolone (followed by oral prednsiolone). One year on follow up, his visual acuity has improved to $6 / 60$ in both eyes. Repeat FFA revealed significant decrease in the non perfused areas of retina.

Conclusions Eye involvement in paediatric lupus is underecognized entity and may need aggressive therapy.

\section{SKIN PEELING AND IRRITABILITY IN A YOUNG BOY WITH SYSTEMIC LUPUS ERYTHEMATOSUS- IS THERE AN OVERLAP WITH KAWASAKI DISEASE?}

A Jindal*, D Suri, A Gupta, A Rawat, S Singh. PGIMER, Paediatrics, chandigarh, India

\subsection{6/lupus-2017-000215.135}

Background and Aims Juvenile systemic lupus erythematosus (SLE) and Kawasaki disease can have several overlapping clinical and laboratory manifestations. But the co-occurrence of both disorders is extremely uncommon.

Methods To report the case of a young boy who had features of both SLE and KD

Results A 5 year old boy presented with fever for 2 months associated with photosensitive malar rash, oral ulcers and myalgias.On examination, he was irritable, had malar rash, a generalised erythematosus macular rash, oral ulcers, red and vertically cracked lips, redness of bulbar conjunctiva, cervical lymphadenopathy and hepatomegaly. Laboratory investigations are summarised in Table 1. All infectious disease workup was normal. He had hypocomplementemia, positive ANA, anti dsDNA and anti-nucleosome antibody. He was diagnosed as SLE and initiated on oral prednisolone. Fever subsided, transaminitis and leucopenia showed gradual recovery. One week later developed periungual skin peeling and he remained irritable. Laboratory investigations showed anaemia, thrombocytosis, persistently elevated ESR and high CRP (Table 1) A diagnosis of incomplete $\mathrm{KD}$ was proffered. $\mathrm{He}$ was given intravenous immunoglobulin (IVIG) (2 gm/ $\mathrm{kg})$, which led to prompt improvement in his irritability. He was initiated on aspirin $(3 \mathrm{mg} / \mathrm{kg} / \mathrm{day})$ and continued on oral prednisolone. Aspirin was discontinued 6 weeks later after confirmation of normal coronary findings on echocardiography. Beau's lines were noticed in the finger nails at this time. At 3 months followup, he continues to remain well. Serum transaminases, platelet counts, ESR and CRP have normalised. (Table 1)

Conclusions Overlap of KD and SLE is extremely uncommon. (Table 2)

Abstract 135 Table 1 Laboratory investigations

\begin{tabular}{|l|l|l|l|l|l|l|l|}
\hline $\begin{array}{l}\text { Investigation } \\
\text { Day of } \\
\text { Admission }\end{array}$ & $\begin{array}{l}\text { Hemoglobin } \\
(\mathrm{gm} / \mathrm{L})\end{array}$ & $\begin{array}{l}\text { White blood cell } \\
\text { count }\left(\times 10^{9} \text { cells } / \mathrm{L}\right)\end{array}$ & $\begin{array}{l}\text { Differential counts } \\
(\mathrm{P} / \mathrm{L} / \mathrm{M} / \mathrm{E})\end{array}$ & $\begin{array}{l}\text { Platelet counts } \\
\left(\times 10^{9} / \mathrm{L}\right)\end{array}$ & $\begin{array}{l}\text { ESR }(\mathrm{mm} \\
\text { in I" hr) }\end{array}$ & $\begin{array}{l}\text { CRP } \\
(\mathrm{mg} / \mathrm{L})\end{array}$ & $\begin{array}{l}\text { AST/ ALT } \\
(\mathrm{U} / \mathrm{L})\end{array}$ \\
\hline Day I & 76 & 3.3 & $30 / 60 / 8 / 2$ & 292 & 52 & 82 & $182 / 73$ \\
\hline Day 5 & 70 & 4.1 & $21 / 69 / 9 / 1$ & 269 & 83 & - & $816 / 287$ \\
\hline Day 9 & 76 & 7.3 & $54 / 38 / 6 / 2$ & 225 & - & - & $456 / 286$ \\
\hline Day 21* & 83 & 14.9 & $45 / 35 / 9$ & 510 & 61 & 16 & $88 / 139$ \\
\hline Day 60 & 100 & 13.6 & $49 / 41 / 9 / 1$ & 400 & 40 & 4 & $36 / 45$ \\
\hline
\end{tabular}

*IVIg was administered on day 21 
Abstract 135 Table 2

\begin{tabular}{|l|l|l|l|}
\hline Sr. no. & Author and year & Age/Sex & Interval between diagnosis of KD and SLE \\
\hline 1 & Laxer et al $(1988)^{4}$ & 5 years $/ \mathrm{F}$ & 3.5 years (KD diagnosed before SLE) \\
\hline 2 & Diniz et al $(2012)^{3}$ & 4 years $/ \mathrm{F}$ & 1 year (KD diagnosed before SLE) \\
\hline 3 & Diniz et al $(2012)^{3}$ & 13 years $/ \mathrm{F}$ & Simultaneous \\
\hline 4 & Present case $(2016)$ & 5 years $/ \mathrm{M}$ & Simultaneous \\
\hline
\end{tabular}

Abbreviations- KD- Kawasaki disease; SLE- systemic lupus erythematosus

136 NEUROPSYCHIATRIC LUPUS WITH HEPATITIS IN A CHILD WITH CMV CO-INFECTION: IS CMV A SIMPLE BYSTANDER?

A Jindal*, S Bhattad, P Vignesh, A Gupta, D Suri, S Singh, A Rawat. PGIMER, Paediatrics, chandigarh, India

10.1136/lupus-2017-000215.136
Background and Aims Cytomegalovirus (CMV) infection at first presentation in paediatric SLE (pSLE) is a rare phenomenon.

Aim- To report an adolescent girl with SLE presenting with neurological and hepatic manifestations and CMV coinfection.

Methods A 12 year old girl presented with history of fever, maculopapular rash over the trunk, malar rash and jaundice.

Abstract 136 Table 1 Laboratory investigations

\begin{tabular}{|l|l|}
\hline Investigation & Result \\
\hline Hemoglobin $(\mathrm{g} / \mathrm{L})$ & 87 \\
\hline Leucocyte counts $(\mathrm{x}$ 109/L) & 6 \\
\hline Platelet counts $(\mathrm{X} 109 / \mathrm{L})$ & 20 \\
\hline Urea/creatinine & $15 / 0.6$ \\
\hline Serum protein/albumin & $5.8 / 2.8$ \\
\hline Total bilirubin/ direct & $13 / 12$ \\
\hline AST/ALT/ALP & $1650 / 120 / 455$ \\
\hline PTI & $50 \%$ \\
\hline aPTT & $57(<28)$ \\
\hline ANA & $3+($ homogenous $)$ \\
\hline Anti-dsDNA & 733 IU/ml $(<40)$ \\
\hline C3 & $68(50-150)$ \\
C4 & $6(20-50)$ \\
\hline DCT & Anti-lgG +,C3d - \\
\hline APL work up & negative \\
\hline Serologies for HAV, HCV, HEV, HIV, EBV & negative \\
\hline CMV PCR & 8160 copies/ml \\
\hline Anti-LKM/ SMA/PCA & negative \\
\hline MRI Brain & Cerebral atrophy \\
\hline CSF examination & $\begin{array}{l}\text { No evidence of meningitis } \\
\text { HSV DNA PCR. CMV PCR - negative }\end{array}$ \\
\hline
\end{tabular}

(DCT - Direct Coombs test, APL- anti-phospholipid, HAV- Hepatitis A, HCV- Hepatitis C, HEV- Hepatitis E, HIV- Human Immunodeficiency Virus, EBV - Epstein Barr Virus) 